\title{
CONTROL OF A NON-HOLONOMIC MOBILE ROBOT SYSTEM WITH PARAMETRIC UNCERTAINTY
}

\author{
Hassan ZARABADIPOUR, Zahra YAGHOUBI
}

\begin{abstract}
In this paper, the control of a mobile robot system via a feedback linearization controller and anti-control of chaos with parametric uncertainty is researched. Anti-control is also applied to convert non-chaotic systems to chaotic ones and to create chaos dynamic. The synchronization of system errors with a chaotic gyroscope system is researched for energy reduction and performance improvement. In the other words, control effort is based on synchronizing the error system with chaos for decreasing control cost. The combination of these techniques yields high efficiency and global convergence of trajectories, even in the presence of parametric uncertainty, which has been shown by simulation. Finally, the energy of control signals is calculated and compared for showing the energy reduction.
\end{abstract}

Keywords: anti-control of chaos; feedback linearization controller; non-holonomic mobile robot; parametric uncertainty

\section{INTRODUCTION}

Recently, the controller of non-holonomic systems became a challenging area for engineers; however, a lot of work has been done in this field. Non-holonomic systems have non-holonomic constraints on velocities. Constraints are considered in wheeled mobile robots [1]. The control of a non-holonomic system is more difficult than that of a holonomic system. Non-holonomic control problems are studied in [2]. The time-varying adaptive control based on backstepping for uncertain non-holonomic chained systems was studied in [3]. There are two approaches for mobile robot control: one of them is stabilization and the other is trajectory tracking, which means the control of robots to track a desired trajectory. Many researchers pay attention to tracking [4-6]. The Lyapunov function is used for designing a tracking controller in [2]. Feedback linearization for tracking is studied in [7], backstepping in [8-9], the sliding mode control in [10], adaptive control in [11], fuzzy control in [12, 13], neural network control in [14], etc.

The mechanical system is synchronized with a chaotic system to control the system in [15]. The chaotification or anti-control of chaos means that a non-chaotic system is to be chaotified. Recently, the application of chaos in control attracts a lot of attention. Some features, such as initial conditions and limitations, are creating complexity in chaotic systems.

Due to the global stability of the chaos system, it is used in this paper, and errors of the states of the system are synchronized with a small ratio of the chaotic system, so that the states of the system can follow the reference path. In this paper, this concept of the anti-control of chaos is used, which refers to the synchronization of the error system with chaotic system.

The gyroscope system is studied in several research papers because of its applications in navigational, aeronautical and space engineering [17, 18]. There are different types of the linear/nonlinear gyroscope which has periodic or chaotic motions $[19,20]$.
The organization of this paper is as follows: in section 2, the chaotic gyroscope system is introduced, in section 3, mobile robot dynamics are described, sections 4 and 5 deal with the feedback linearization controller for nonlinear systems and mobile robot, in section 6 , the control of mobile robot systems with feedback linearization via the anti-control of chaos is researched, in section 7, the linearization controller for the mobile robot with parametric uncertainty is studied, the simulation results are provided in section 8 and finally, the conclusion is provided in the last section.

\section{CHAOTIC GYROSCOPE SYSTEM}

The gyroscope is modelled as follows and the scheme of it is shown in Fig. 1(c) [21]:

$\ddot{\theta}+\alpha_{1}^{2} \frac{(1-\cos \theta)^{2}}{\sin ^{3} \theta}-\beta_{1} \sin \theta+c_{1} \dot{\theta}+c_{2} \dot{\theta}^{3}=f \sin \omega t \sin \theta$

Where $f \sin \omega t$ is defined as parametric excitation, the linear and nonlinear damping terms are $c_{1} \dot{\theta}$ and, $c_{2} \dot{\theta}^{3}$ and $\alpha_{1}^{2} \frac{(1-\cos \theta)^{2}}{\sin ^{3} \theta}-\beta_{1} \sin \theta$ is a nonlinear force.

By giving $x_{1}=\theta, \quad x_{2}=\dot{\theta}$ and $g(\theta)=\alpha_{1}^{2} \frac{(1-\cos \theta)^{2}}{\sin ^{3} \theta}-$ $-\beta \sin \theta$, Eq. (1) can be rewritten as follows:

$\dot{x}_{1}=x_{2}$

$\dot{x}_{2}=-g\left(x_{1}\right)-c_{1} x_{2}-c_{2} x_{2}^{3}+f \sin \omega t \sin \left(x_{1}\right)$

Where the parameters are defined as follows: $32<f<36$, $\alpha_{1}^{2}=100, \beta_{1}=1, c_{1}=0.5, c_{2}=0.05$, and $\omega=2$. Fig. 1(a) and 
1(b) illustrates the irregular motion exhibited by this system for $f=35.5$ and the initial conditions of $\left(x_{1}, x_{2}\right)=(1,-1)$.
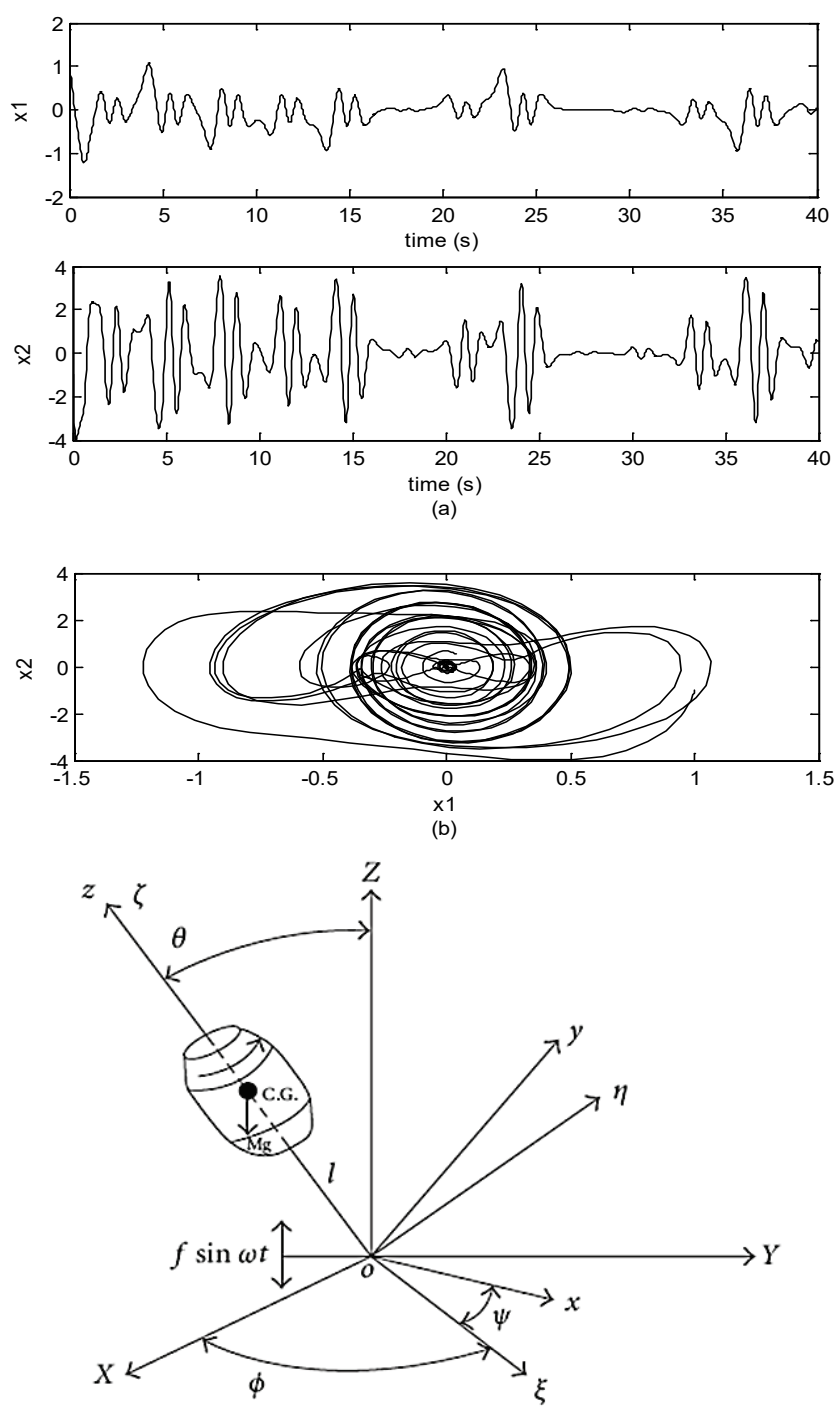

(c)

Figure 1 (a) Time series of $x 1$ and $x 2$, (b) Phase Portrait of a gyroscope, (c) A schematic diagram of a symmetric gyroscope

\section{MOBILE ROBOT KINEMATICS AND DYNAMICS}

The mobile robot system is modelled as follows:

$$
\left[\begin{array}{c}
\dot{x} \\
\dot{y} \\
\dot{\theta}
\end{array}\right]=\left[\begin{array}{cc}
\cos \theta & 0 \\
\sin \theta & 0 \\
0 & 1
\end{array}\right] \cdot\left[\begin{array}{l}
v \\
\omega
\end{array}\right]
$$

Where the control inputs of $v$ and $\omega$ are the forward and angular velocities, as shown in Fig. 2(a). The nonholonomic constraint, which comes from the non-slip condition, is described as follows [24]:

$\dot{x} \sin \theta+\dot{y} \cos \theta=0$
Let us assume that $\left(x_{r}, y_{r}, \theta_{r}, v_{r}, \omega_{r}\right)^{\mathrm{T}}$ are the references, hence the following equations are satisfied:

$$
\left[\begin{array}{l}
\dot{x}_{r} \\
\dot{y}_{r} \\
\dot{\theta}_{r}
\end{array}\right]=\left[\begin{array}{cc}
\cos \theta_{r} & 0 \\
\sin \theta_{r} & 0 \\
0 & 1
\end{array}\right] \cdot\left[\begin{array}{c}
v_{r} \\
\omega_{r}
\end{array}\right]
$$

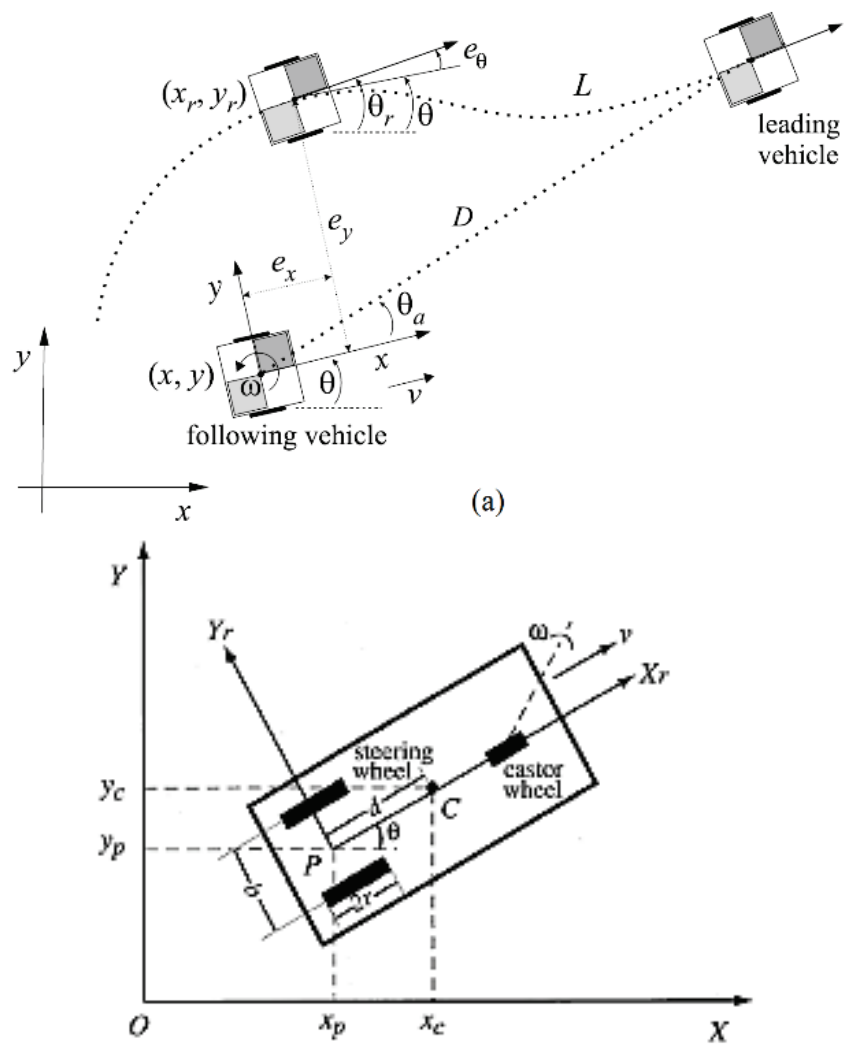

(b)

Figure 2 (a) Illustration of error transformation where the following vehicle follows the path of the leading vehicle at the distance L [24]; (b) Mobile robot with two actuated wheels

Where the control inputs references are derived as follows:

$$
v_{r}=\sqrt{\dot{x}_{r}^{2}+\dot{y}_{r}^{2}} \quad, \quad \omega_{r}=\frac{\dot{x}_{r} \ddot{y}_{r}-\dot{y}_{r} \ddot{x}_{r}}{\dot{x}_{r}^{2}+\dot{y}_{r}^{2}}
$$

Fig. 2(a) shows the error when the real vehicle follows the reference vehicle. The error system is described as follows [24]:

$$
e=\left[\begin{array}{c}
e_{x} \\
e_{y} \\
e_{\theta}
\end{array}\right]=\left[\begin{array}{ccc}
\cos \theta & \sin \theta & 0 \\
-\sin \theta & \cos \theta & 0 \\
0 & 0 & 1
\end{array}\right] \cdot\left[\begin{array}{c}
x_{r}-x \\
y_{r}-y \\
\theta_{r}-\theta
\end{array}\right]
$$

By differentiating Eq. (7), the nonlinear error model of the system is obtained as follows: 


$$
\left[\begin{array}{c}
\dot{e}_{x} \\
\dot{e}_{y} \\
\dot{e}_{\theta}
\end{array}\right]=\left[\begin{array}{c}
v_{r} \cos e_{\theta} \\
v_{r} \sin e_{\theta} \\
\omega_{r}
\end{array}\right]+\left[\begin{array}{cc}
-1 & e_{y} \\
0 & -e_{x} \\
0 & -1
\end{array}\right]\left[\begin{array}{l}
v \\
\omega
\end{array}\right]
$$

For the dynamic model, Eq. (9) is added to Eq. (3):

$$
\left\{\begin{array}{l}
\dot{v}=\frac{1}{m r}\left(\tau_{1}+\tau_{2}\right) \\
\dot{\omega}=\frac{b}{2 I r}\left(\tau_{1}-\tau_{2}\right)
\end{array}\right.
$$

Where $m$ is the mass and $I$ the moment inertia of mobile robot with the radius of the rear wheels $r$ and the length the between wheels $b, \tau_{1}$ and $\tau_{2}$ are the control torques of each rear wheel as it is shown in Fig. 2(b).

The error $\left(e_{x}, e_{y}, e_{\theta}\right)^{T}$ converges to zero with control laws for $v$ and $\omega$, and this is a tracking control problem.

\section{FEEDBACK LINEARIZATION CONTROLLER FOR MIMO NONLINEAR SYSTEMS}

The general system is considered as follows:

$$
\begin{aligned}
& \left\{\begin{array}{l}
\dot{x}=f(x)+\sum_{i=1}^{m} g_{i} \cdot u_{i} \\
y=\left[h_{1}, \ldots, h_{m}\right]^{T}
\end{array}\right. \\
& \dot{y}_{k}=L_{f}\left(h_{k}\right)+\sum_{i=1}^{m} L_{g_{i}}\left(h_{k}\right) u_{i}
\end{aligned}
$$

$r_{k}$ is the relative degree and $L_{f}(h)$ is the lie bracket $[f, h]$.

For some $i, L_{g_{i}}\left(L_{f}^{r_{k}-1}\left(h_{k}\right)\right) \neq 0$.

$J(x)$ is the decoupling matrix and the $m \times m$ matrix is defined as follows:

$$
J(x)=\left[\begin{array}{cccc}
L_{g_{1}}\left(L_{f}^{r_{1}-1}\left(h_{1}\right)\right) & \ldots & L_{g_{m}}\left(L_{f}^{r_{1}-1}\left(h_{1}\right)\right) \\
\ldots & & \ldots & \ldots \\
L_{g_{1}}\left(L_{f}^{r_{m}-1}\left(h_{m}\right)\right) & \ldots & L_{g_{m}}\left(L_{f}^{r_{m}-1}\left(h_{m}\right)\right)
\end{array}\right]
$$

Where $r_{k}$ is the relative degree, $L_{f}(h)$ is the lie bracket $[f, h]$ and $J(x)$ is assumed to be non-singular.

Let the $m \times 1$ be a vector $y^{r}$ :

$$
\begin{gathered}
y^{r}=\left[\begin{array}{c}
\frac{d^{r_{1}} y_{1}}{d t^{r_{1}}} \\
\ldots \\
\frac{d^{r_{m}} y_{m}}{d t^{r_{m}}}
\end{array}\right] \\
l(x)=\left[\begin{array}{c}
L_{f}^{r_{1}}\left(h_{1}\right) \\
\ldots \\
L_{f}^{r_{m}}\left(h_{m}\right)
\end{array}\right]
\end{gathered}
$$

Therefore,

$$
y^{r}=l(x)+J(x), u=v
$$

Where $l(x)$ is the $m \times 1$ vector and $v$ is the $m \times 1$ input. A decoupled set of equations are given as follows:

$$
\left\{\begin{array}{l}
\frac{d^{r_{1}} y_{1}}{d t^{r_{1}}}=v_{1} \\
\cdots \\
\frac{d^{r_{m}} y_{m}}{d t^{r_{m}}}=v_{m}
\end{array} \text { so } \quad y \Leftrightarrow v\right.
$$

The input $v$ is designed by methods such as: pole placement method, LQR, etc. For example,

$$
\begin{aligned}
& v=-c_{0} y-c_{1} \dot{y}-c_{2} \ddot{y} \ldots \\
& \Rightarrow y^{(r)}+c_{r-1} y^{(r-1)}+\ldots+c_{0} y=0
\end{aligned}
$$

The control, $u$, is given as:

$$
u=J^{-1}(v-l)
$$

\section{FEEDBACK LINEARIZATION CONTROLLER FOR MOBILE ROBOT}

The feedback linearization controller will be designed for the system described by Eq. (3), as shown in Fig. 3.

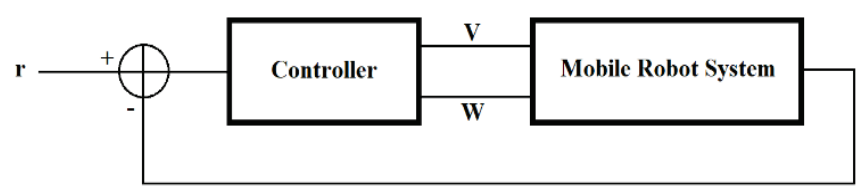

Figure 3 Block diagram of the control system.

If we define $y_{1}=x_{1}$ and $y_{2}=x_{2}$ as outputs, then we have: 
$\left[\begin{array}{l}\dot{y}_{1} \\ \dot{y}_{2}\end{array}\right]=\left[\begin{array}{ll}\cos \theta & 0 \\ \sin \theta & 0\end{array}\right]\left[\begin{array}{l}v \\ \omega\end{array}\right]$

$J(x)=\left[\begin{array}{cc}\cos \theta & 0 \\ \sin \theta & 0\end{array}\right]$ is singular and its rank is one.

Let us suppose that $v=x_{3}, \dot{v}=\dot{x}_{3}=u_{1}, \omega=u_{2}$.

The states have been extended as:

$\left\{\begin{array}{l}\dot{x}_{1}=x_{3} \cos \theta \\ \dot{x}_{2}=x_{3} \sin \theta \\ \dot{x}_{3}=\dot{v}=u_{1} \\ \dot{\theta}=\omega=u_{2}\end{array}\right.$

Take $y_{1}=x_{1}$ and $y_{2}=x_{2}$.

$\left[\begin{array}{l}\ddot{y}_{1} \\ \ddot{y}_{2}\end{array}\right]=\left[\begin{array}{cc}\cos \theta & -x_{3} \sin \theta \\ \sin \theta & x_{3} \cos \theta\end{array}\right]\left[\begin{array}{l}u_{1} \\ u_{2}\end{array}\right]$

The relative degree of the system is 2 and the matrix $J(x)$ is $J(x)=\left[\begin{array}{cc}\cos \theta & -x_{3} \sin \theta \\ \sin \theta & x_{3} \cos \theta\end{array}\right]$, which is non-singular for $x_{3} \neq 0$.

Let us suppose

$\left\{\begin{array}{l}\ddot{y}_{1}=V_{1} \\ \ddot{y}_{2}=V_{2}\end{array}\right.$

The control, $u$, is obtained as:

$\left[\begin{array}{l}u_{1} \\ u_{2}\end{array}\right]=J^{-1}(x)\left[\begin{array}{l}V_{1} \\ V_{2}\end{array}\right]=\left[\begin{array}{cc}\cos \theta & \sin \theta \\ \frac{-1}{x_{3}} \sin \theta & \frac{1}{x_{3}} \cos \theta\end{array}\right]\left[\begin{array}{l}V_{1} \\ V_{2}\end{array}\right]$

Now $\left(V_{1}, V_{2}\right)^{\mathrm{T}}$ can be obtained by using the pole placement technique in Eq. (17) and $\dot{v}=u_{1}$.

It should be noted that, by using the pole placement method, the mobile robot converges faster or more slowly to the desired path depending on the occasion of poles.

\section{FEEDBACK LINEARIZATION CONTROLLER FOR A MOBILE ROBOT BY USING THE ANTI-CONTROL OF CHAOS (CHAOTIFICATION)}

In this section, the errors of states track the chaotic gyroscope system with a small amplitude, but in section 5, error signals are converged to zero, as shown in Fig. 4 . The coefficient $\alpha$ is used to reduce the magnitude of chaotic systems and $10^{-10} \leq \alpha \leq 1$.

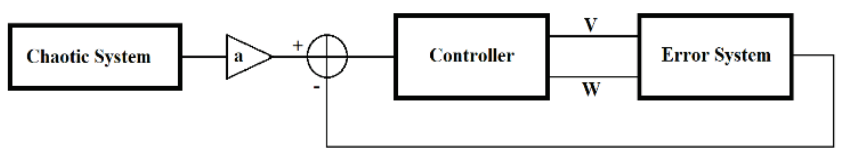

Figure 4 Block diagram of the control system

Thus, the error system in Eq. (8) is studied by using the feedback linearization technique. $y_{1}=e_{y}$ and $y_{2}=e_{\theta}$ are defined as outputs.

$\left[\begin{array}{l}\dot{y}_{1} \\ \dot{y}_{2}\end{array}\right]=\left[\begin{array}{cc}0 & -e_{x} \\ 0 & -1\end{array}\right]\left[\begin{array}{l}v \\ \omega\end{array}\right]+\left[\begin{array}{c}v_{r} \sin e_{\theta} \\ \omega_{r}\end{array}\right]$

$J(x)=\left[\begin{array}{cc}0 & -e_{x} \\ 0 & -1\end{array}\right]$ with rank one is singular.

Let $v=u_{1}, \omega=x_{4}, \quad \dot{\omega}=\dot{x}_{4}=u_{2}$.

The states have been extended as:

$\left\{\begin{array}{l}\dot{e}_{x}=v_{r} \cos e_{\theta}-u_{1}+e_{y} x_{4} \\ \dot{e}_{y}=v_{r} \sin e_{\theta}-e_{x} x_{4} \\ \dot{e}_{\theta}=\omega_{r}-x_{4} \\ \dot{x}_{4}=\dot{\omega}=u_{2}\end{array}\right.$

Assuming that $y_{1}=e_{y}$ and $y_{2}=e_{\theta}$.

$\left[\begin{array}{l}\ddot{y}_{1} \\ \ddot{y}_{2}\end{array}\right]=\left[\begin{array}{cc}x_{4} & -e_{x} \\ 0 & -1\end{array}\right]\left[\begin{array}{l}u_{1} \\ u_{2}\end{array}\right]+\left[\begin{array}{c}\left(-2 v_{r} x_{4}+v_{r} \omega_{r}\right) \cos e_{\theta}-e_{y} x_{4}^{2}+\dot{v}_{r} \sin e_{\theta} \\ \dot{\omega}_{r}\end{array}\right]$

The relative degree of the system is 2 and the matrix $J(x)$ is $J(x)=\left[\begin{array}{cc}x_{4} & -e_{x} \\ 0 & -1\end{array}\right]$ that is non-singular for $x_{4} \neq 0$, and $l(x)=\left[\begin{array}{c}\left(-2 v_{r} x_{4}+v_{r} \omega_{r}\right) \cos e_{\theta}-e_{y} x_{4}^{2}+\dot{v}_{r} \sin e_{\theta} \\ \dot{\omega}_{r}\end{array}\right]$

The control, $u$, is obtained by using Eq. (22):

$\left[\begin{array}{l}u_{1} \\ u_{2}\end{array}\right]=J^{-1}(x)\left(\left[\begin{array}{l}V_{1} \\ V_{2}\end{array}\right]+l(x)\right)=$
$=\left[\begin{array}{c}\left.\frac{1}{x_{4}}\left(V_{1}+\left(2 v_{r} x_{4}-v_{r} \omega_{r}\right) \cos e_{\theta}+e_{y} x_{4}^{2}-\dot{v}_{r} \sin e_{\theta}-e_{x}\left(V_{2}-\dot{\omega}_{r}\right)\right)\right] \\ -\left(V_{2}-\dot{\omega}_{r}\right)\end{array}\right]$

Designing $\left(V_{1}, V_{2}\right)^{T}$ by using pole placement technique in Eq. (17) and $\dot{\omega}=u_{2}$. 


\section{FEEDBACK LINEARIZATION CONTROLLER FOR A MOBILE ROBOT WITH PARAMETRIC UNCERTAINTY}

In this section, nonlinear control laws are proposed for the control of the mobile robot motion described by Eq. (3) and Eq. (9). Feedback control for $\tau_{1}$ and $\tau_{2}$ in the presence of uncertainty in the parameter $m$ is derived.

Assuming that $y_{1}=x$ and $y_{2}=\theta$. The relative degrees are 2 and the control signals without the anti-control of chaos are obtained as:

$\left[\begin{array}{c}\tau_{1} \\ \tau_{2}\end{array}\right]=\left[\begin{array}{l}\frac{r m}{2 \cos \theta}\left(V_{1}+v \omega \sin \theta\right)+\frac{r I}{b} V_{2} \\ \frac{r m}{2 \cos \theta}\left(V_{1}+v \omega \sin \theta\right)-\frac{r I}{b} V_{2}\end{array}\right]$
The control signals with the anti-control of chaos with the Eqs. (8) and (9) are obtained as Eq. (29):

$$
\left[\begin{array}{l}
\tau_{1} \\
\tau_{2}
\end{array}\right]=\left[\begin{array}{l}
r m \\
-\frac{r m}{2}\left(V_{1}-\dot{v}_{r} \cos e_{\theta}+v_{r} \omega_{r} \sin e_{\theta}-2 v_{r} \omega \sin e_{\theta}+\omega^{2} e_{x}\right)-\left(\frac{r I}{b}+\frac{r m}{2} e_{y}\right)\left(V_{2}-\dot{\omega}_{r}\right) \\
-\frac{r m}{2}\left(V_{1}-\dot{v}_{r} \cos e_{\theta}+v_{r} \omega_{r} \sin e_{\theta}-2 v_{r} \omega \sin e_{\theta}+\omega^{2} e_{x}\right)+\left(\frac{r I}{b}-\frac{r m}{2} e_{y}\right)\left(V_{2}-\dot{\omega}_{r}\right)
\end{array}\right]
$$

\section{SIMULATION RESULTS \\ 8.1 Control of the Mobile Robot}

Feedback linearization control is applied to a mobile robot system with a different desired reference and the results are shown in Fig. 5. Reference paths are Circle (circular reference: $\left(x_{r}=\cos t, y_{r}=\sin t\right)$, Sine wave $\left(x_{r}=t, y_{r}=\sin t\right)$ and Sinc wave $\left(x_{r}=t, y_{r}=\sin t / t=\operatorname{sinc} t\right)$. In these cases, poles $=-100$ were chosen for the linear controller.
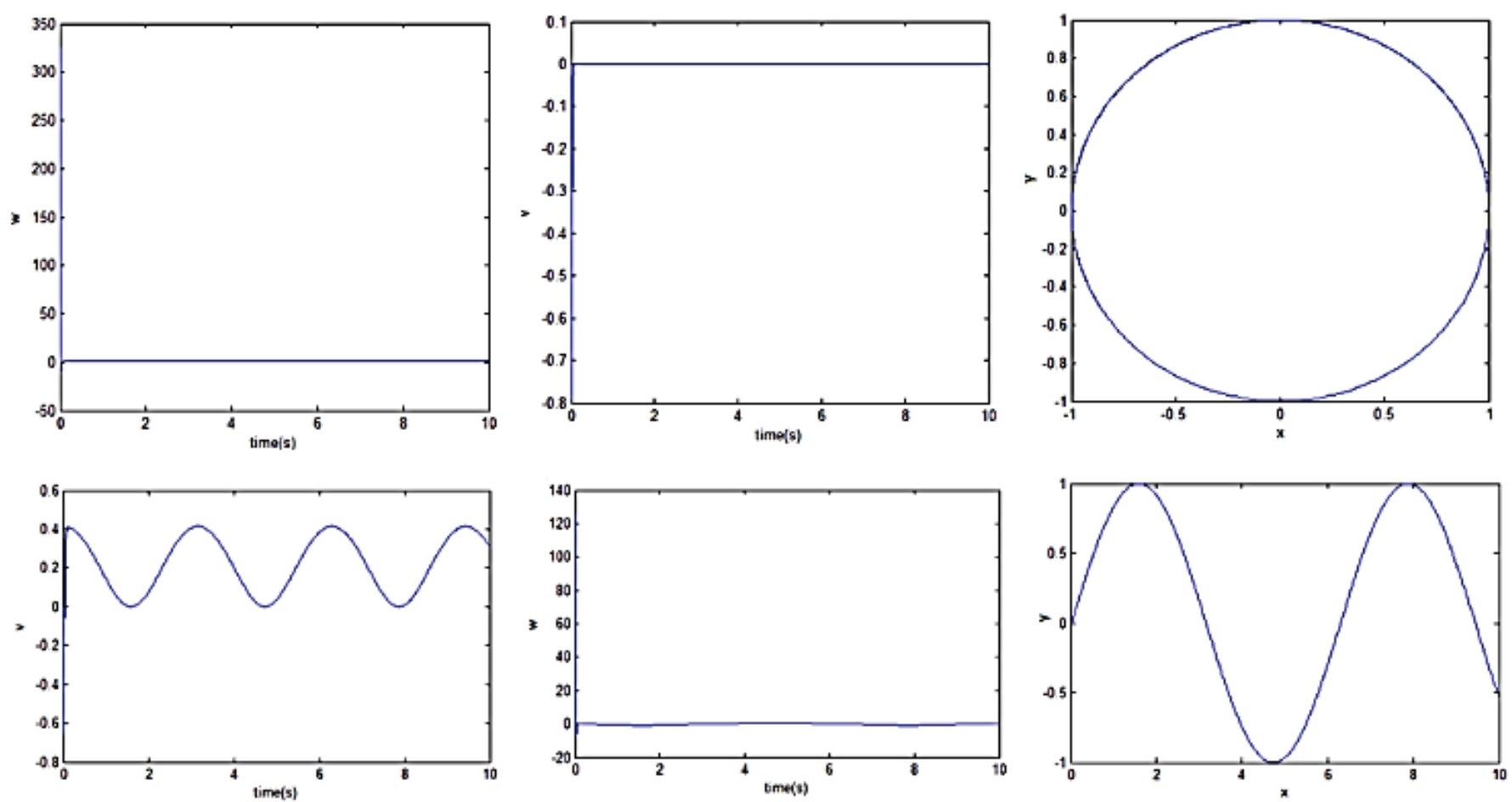

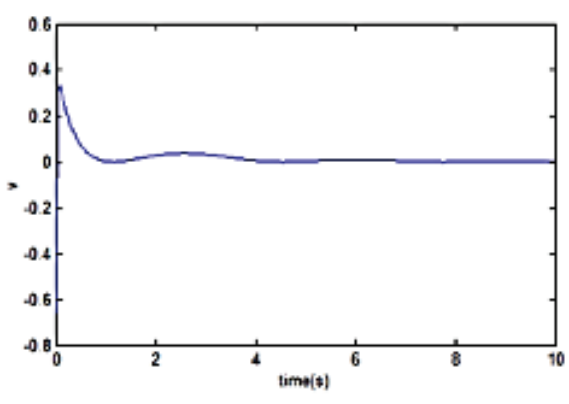

(a)

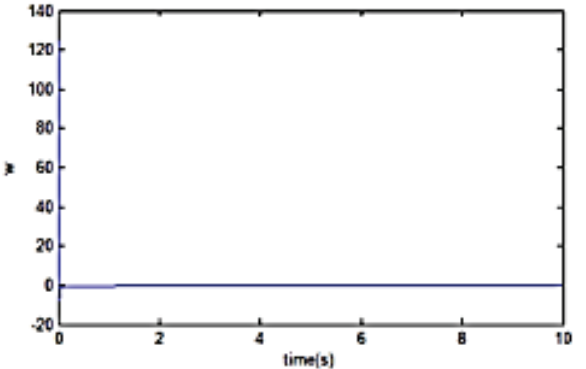

Figure 5 Control signals for references and path following

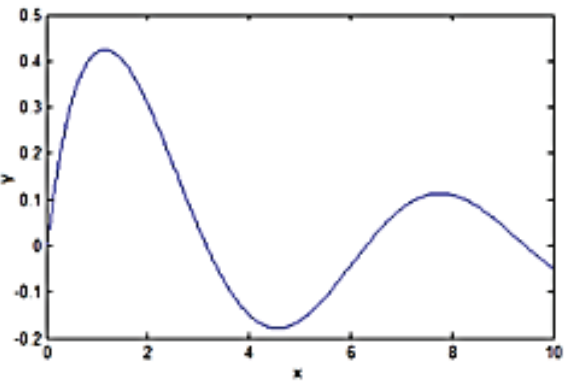

(b) 

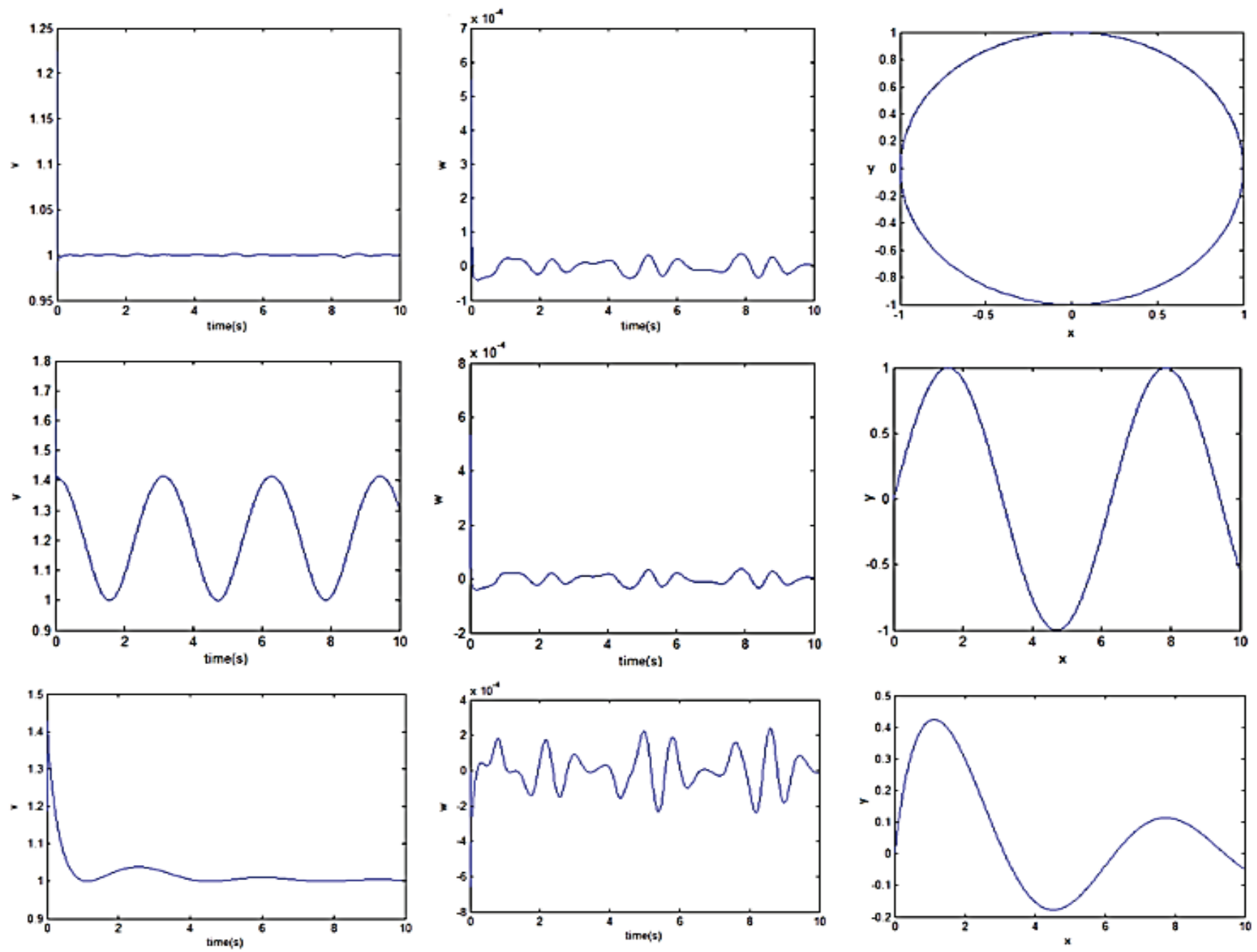

(a)

Figure 6 Control signals for references and path following

(b)

\subsection{Anti-control of Chaos for Controlling the Mobile Robot}

The mobile robot system is controlled via the anticontrol of chaos with a different desired reference, and they are shown in Figure 6. In these cases, poles $=-150$ were chosen for the linear controller. As shown in Fig. 4, the two states of the mobile robot system and the error system are synchronized with the two states of chaotic gyroscope systems.

\subsection{Mobile Robot Control with Parametric Uncertainty}

In this section, the performance of the feedback linearization controller with parametric uncertainty is illustrated. The parameters were adopted as follows for the circle reference: $m=10 \mathrm{~kg}, I=1.25 \mathrm{kgm}^{2}, b=1 \mathrm{~m}$ and $r=$ $0.1 \mathrm{~m}$.

The parameter $m$ with uncertainty is in Fig. 7.(a), a poor tracking of variables $x$ and $y$ by using the feedback linearization controller in the presence of parametric uncertainty is shown, while in Fig. 7(d), the feedback linearization controller with the anti-control of chaos was applied to better tracking. Fig. 7(b) and 7(c) shows control signals and 7(d) shows the tracking comparison of the feedback linearization controller with the anti-control of chaos, whether in the presence of parametric uncertainty or not.

\subsection{Energy Comparison}

In this paper, the main purpose is the reduction of energy or control cost with synchronization error system with chaos system. The energy is defined as follows, which it means the integral of signals squared (area under the curve):

$$
\text { energy }=\sum\left(v^{2}+\omega^{2}\right)
$$

The energy comparison is shown in Tab. 1. It is shown that the anti-control of chaos causes the energy reduction.

Table 1 Energy comparison.

\begin{tabular}{|l|c|c|c|}
\hline \multicolumn{1}{|c|}{ Control } & circle & sine wave & sinc wave \\
\hline feedback linearization control & 15683 & 31415 & 30890 \\
\hline anti-control of chaos & 592.08 & 818.55 & 565.29 \\
\hline
\end{tabular}



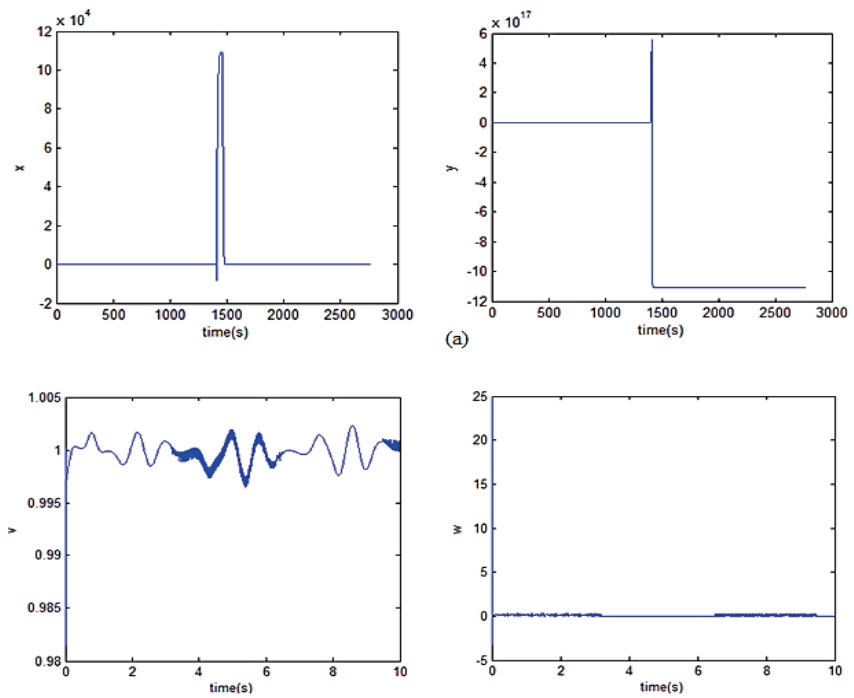

(b)

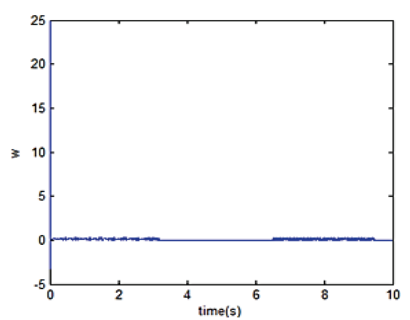

(c)

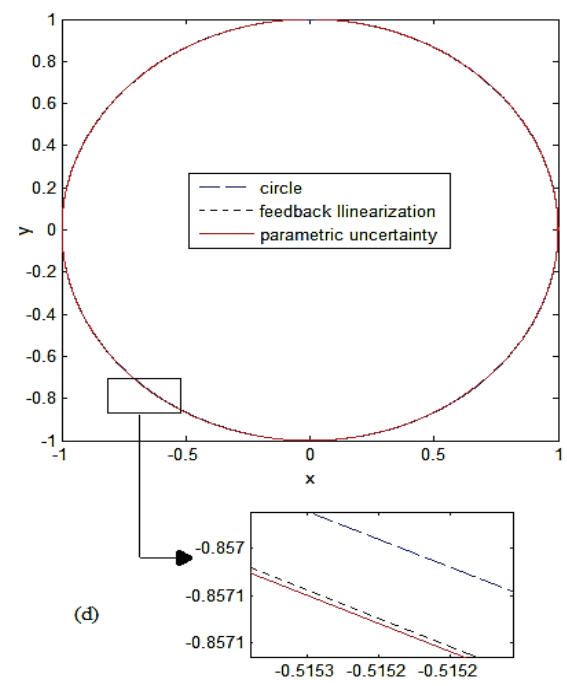

Figure 7 Path following in the presence of parametric uncertainty. (a) Using the feedback linearization controller. Feedback linearization controller with the anticontrol of chaos: (b) Control signal (v), (c) Control signal (w), (d) Path following

\section{CONCLUSION}

In this paper, the control of the mobile robot system has been researched in two different ways, and the feedback linearization controller has been used in both of them. Different desired references have been applied for a better comparison. Due to the positive feature of chaotic systems such as energy improvement and control cost, the errors of the states of the mobile robot system are synchronized with the chaotic system.

While the desired tracking error is permanently zero, the control effort is based on the convergence of the error to zero, which is a somewhat strict way of dealing with the issue. The fact is that although by decreasing the error, the desired control is obtained, but too much control cost should be applied, while the anti-control of chaos solves this problem.

The non-linear control laws that yield convergence of the trajectories in the presence of parametric uncertainty have been derived. Simulation results are proposed to illustrate the effectiveness of the proposed controller.

\section{REFERENCES}

[1] Bloch, A. M., Baillieul, J., Crouchand P., \& Marsden, J. E. (2003). Nonholonomic mechanics and control. New York, Berlin, Heidelberg. https://doi.org/10.1007/b97376

[2] Kanayama, Y., Kimura, Y., Miyazakiand, F., \& Noguchi, T. (1990). A Stable Tracking Control Method for an Autonomous Mobile Robot. IEEE International Conference on Robotics and Automation, 384-389. https://doi.org/10.1109/ROBOT.1990.126006

[3] Jiang, Z. P. \& Pomet, J. B. (1996). Global stabilization of parametric chained systems by time-varying dynamic feedback. International Journal of Adaptive Control and Signal Processing, 10, 47-59. https://doi.org/10.1002/(SICI)1099-1115(199601)10:1<47::AIDACS385>3.0.CO;2-7

[4] Dierks, T. \& Jagannathan, S. (2009). Asymptotic Adaptive Neural Network Tracking Control of Nonholonomic Mobile Robot Formations. Journal of Intelligent and Robotic Systems, 56(1), 153-176. https://doi.org/10.1007/s10846-009-9336-8

[5] Lee, J. H., Lin, C., Lim, H., \& Lee, J. M. (2009). Sliding Mode Control for Trajectory Tracking of Mobile Robot in the RFID Sensor Space. IJCAS, 7(3), 429-435. https://doi.org/10.1007/s12555-009-0312-7

[6] Michalek, M., Dutkiewicz, P., Kielczewski, M., \& Pazderski, D. (2009). Trajectory Tracking for a Mobile Robot with SkidSlip Compensation in the Vector-Field-Orientation Control System. AMCS, 19(4), 547-559. https://doi.org/10.2478/v10006-009-0043-1

[7] d'Andrea-Novel, B., Campion, G., \& Bastin, G. (1995). Control of a nonholonomic wheeled mobile robot by state feedback linearization. IJRR, 14(6), 543-559. https://doi.org/10.1177/027836499501400602

[8] Jinag, Z. P. \& Nijmeijer, H. (1997). Tracking Control of Mobile Robots: A Case Study in Backstepping. Automatica, 33, 1393-1399.

[9] Lee, T. C. \& Tai, K. (2011). Tracking Control of UnicycleModeled Mobile robots using a Saturation Feedback Controller. IEEE T CONTR SYST T., 9(2), 305-318.

[10] Yang, J. M. \& Kim, J. H. (1999). Sliding Mode Control for Trajectory of Nonholonomic Wheeled Mobile Robots. IEEE T$R O, 15(3), 578-587$.

[11] Pourboghrat, F. \& Karlsson, M. P. (2002). Adaptive Control of Dynamic Mobile Robots with Nonholonomic Constraints. Computers and Electrical Engineering, 28, 241-253. https://doi.org/10.1016/S0045-7906(00)00053-7

[12] Imen, M., Mansouri, M., \& Aliyari, Sh. M. (2011). Tracking Control of Mobile Robot Using ANFIS. IEEE International Conference on Mechatronics and Automation, August 7-10, Beijing, China, 422-427. https://doi.org/10.1109//CMA.2011.5985695

[13] Wang, S. D. \& Lin, C. K. (2000). Adaptive Tuning of the Fuzzy Controller for Robots. Fuzzy Sets Systems, 110(2), 351-363. https://doi.org/10.1016/S0165-0114(98)00078-5

[14] Yildirim, S. (2004). Adaptive Robust Neural Controller for Robots. Robotics and Autonomous Systems, 46(1), 175-184. https://doi.org/10.1016/j.robot.2003.11.008

[15] Chen, H. K. \& Lee, Ch. I. (2004). Anti-control of chaos in rigid body motion. CS\&F, 21, 957-965. https://doi.org/10.1016/j.chaos.2003.12.034 
[16] Chen, G. \& Dong, X. (1988). From chaos to order: Perspectives, methodologies and applications. World Scientific, Singapore, 50-51.

[17] Chen, H. K. (2002). Chaos and chaos synchronization of a symmetric gyro with linear-plus-cubic damping. JSV, 255(4), 719-740. https://doi.org/10.1006/jsvi.2001.4186

[18] Farivar, F., Aliyari, Sh, M., Nekoui, M. A., \& Teshnehlab, M. (2012). Chaos control and generalized projective synchronization of heavy symmetric chaotic gyroscope systems via Gaussian radial basis adaptive variable structure control. $C S \& F, 45,80-97$.

https://doi.org/10.1016/j.chaos.2011.10.008

[19] Ge, Z. M. (2002). Chaos control for rigid body systems. Taipei. GauLih Book Company.

[20] Lei, Y., Xu, W., \& Zheng, H. (2005). Synchronization of two chaotic nonlinear gyros using active control. Physics Letters A. 343, 153-158. https://doi.org/10.1016/j.physleta.2005.06.020

[21] Farivar, F., Aliyari, Sh. M., Nekoui, M. A., \& Teshnehlab, M. (2009). Chaos synchronization of uncertain nonlinear gyros via hybrid control. IEEE/ASME international conference on advanced intelligent mechatronics, 1365-1370. https://doi.org/10.1109/AIM.2009.5229879

[22] Yau, H. T. (2008). Synchronization and anti-synchronization coexist in twodegree- of-freedom dissipative gyroscope with nonlinear inputs. Nonlinear Analysis Real World Applications, 9(5), 2253-2261. https://doi.org/10.1016/j.nonrwa.2007.08.002

[23] Van, D. R. \& Chen, H. K. (2003). Chaos and chaos synchronization of a symmetric gyro with linear-plus-cubic damping. JSV, 268(3), 632-636. https://doi.org/10.1016/S0022-460X(03)00343-2

[24] Klančar, G., Matko, D., \& Blažič, S. (2010). A control strategy for platoons of differential drive wheeled mobile robot. RAS, $59,57-64$.

\section{Authors' contacts:}

Hassan ZARABADIPOUR, PhD, Associate Professor Department of Electrical Engineering Imam Khomeini International University

Qazvin, Iran

hzarabadi@eng.kiku.ac.ir

\section{Zahra YAGHOUBI, M.Sc.}

Department of Electrical Engineering Imam Khomeini International University, Qazvin, Iran

z_yaghoubi@aut.ac.ir 